ISSN 2590-9770

The Art of Discrete and Applied Mathematics 3 (2020) \#P1.02

https://doi.org/10.26493/2590-9770.1291.c54

(Also available at http://adam-journal.eu)

\title{
On strongly sequenceable abelian groups
}

\author{
Brian Alspach (D), Georgina Liversidge \\ School of Mathematical and Physical Sciences, University of Newcastle, \\ Callaghan, NSW 2308, Australia
}

Received 4 February 2019, accepted 7 August 2019, published online 22 July 2020

\begin{abstract}
A group is strongly sequenceable if every connected Cayley digraph on the group admits an orthogonal directed cycle or an orthogonal directed path. This paper deals with the problem of whether finite abelian groups are strongly sequenceable. A method based on posets is used to show that if the connection set for a Cayley digraph on an abelian group has cardinality at most nine, then the digraph admits either an orthogonal directed path or an orthogonal directed cycle.
\end{abstract}

Keywords: Strongly sequenceable, abelian group, diffuse poset, sequenceable poset.

Math. Subj. Class. (2020): 05C25

\section{Introduction}

The Cayley digraph $\overrightarrow{\mathrm{Cay}}(G ; S)$ on the group $G$ has the elements of $G$ for the vertex set and an $\operatorname{arc}(g, h)$ from $g$ to $h$ whenever $h=g s$ for some $s \in S$, where $S \subset G$ and $1 \notin S$. The set $S$ is called the connection set. It is easy to see that left-multiplication by any element of $G$ is an automorphism of $\overrightarrow{\mathrm{Cay}}(G ; S)$ which implies that the automorphism group of $\overrightarrow{\mathrm{Cay}}(G ; S)$ contains the left-regular representation of $G$.

A given $s \in S$ generates a spanning digraph of $\overrightarrow{\mathrm{Cay}}(G ; S)$ composed of vertex-disjoint directed cycles of length $|s|$, where $|s|$ denotes the order of $s$. We call this subdigraph a $(1,1)$-directed factor because the in-valency and out-valency at each vertex is 1 . Hence, there is a natural factorization of $\overrightarrow{\mathrm{Cay}}(G ; S)$ into $|S|$ arc-disjoint $(1,1)$-directed factors. This is the Cayley factorization of $\overrightarrow{\mathrm{Cay}}(G ; S)$ and is denoted $\mathcal{F}(G ; S)$.

Let $\overrightarrow{\mathrm{Cay}}(G ; S)$ be a Cayley digraph on a group $G$. A subdigraph $\vec{Y}$ of $\overrightarrow{\mathrm{Cay}}(G ; S)$ of size $|S|$ (the size is the number of arcs in $\vec{Y}$ ), is orthogonal to $\mathcal{F}(G ; S)$ if $\vec{Y}$ has one arc

E-mail addresses: brian.alspach@newcastle.edu.au (Brian Alspach), gliv560@aucklanduni.ac.nz (Georgina Liversidge)

(a)(i) This work is licensed under https://creativecommons.org/licenses/by/4.0/ 
from each $(1,1)$-directed factor of $\mathcal{F}(G ; S)$. In order to simplify the language, we simply say that $\overrightarrow{\mathrm{Cay}}(G ; S)$ admits an orthogonal $\vec{Y}$.

The complete digraph $\vec{K}_{n}$ may be viewed as a Cayley digraph $\vec{K}(G)$ on any group $G$ of order $n$ by choosing the connection set to be $G \backslash\{1\}$. B. Gordon [13] defined a group $G$ to be sequenceable if $\vec{K}(G)$ admits an orthogonal Hamilton directed path (he used different language). Gordon was motivated by looking for methods to produce row-complete Latin squares and a sequenceable group gives rise to a row-complete Latin square.

From his work on the Heawood map coloring problem, G. Ringel [18] asked when does $\vec{K}(G)$ admit an orthogonal directed cycle of length $|G|-1$ (he also used different language). A group $G$ for which this holds was called $R$-sequenceable in [12].

So the two notions of a sequenceable group and an $R$-sequenceable group were motivated by quite disparate mathematical problems, but as we have seen they are closely related. The topic of sequenceable and $R$-sequenceable groups has generated, and continues to generate, a considerable amount of research. There have been surveys $[11,17]$ and many papers including $[1,2,3,4,5,6,7,12,13,14,16]$.

The following definition is a natural extension of sequenceable and $R$-sequenceable groups.

Definition 1.1. A group $G$ is strongly sequenceable if every connected Cayley digraph on $G$ admits either an orthogonal directed path or an orthogonal directed cycle.

An abelian group cannot be both sequenceable and $R$-sequenceable, but by allowing either an orthogonal directed path or an orthogonal directed cycle in the definition of strongly sequenceable, we guarantee that when an abelian group is strongly sequenceable, it is either sequenceable or $R$-sequenceable.

The first author and T. Kalinowski have posed the following problem.

Research problem 1.2. Determine the strongly sequenceable groups.

\section{Abelian groups}

It is not difficult to verify that the non-abelian group of order 6 is not strongly sequenceable. The only connection set for which it fails is the one giving $\vec{K}_{6}$.

There has been some work on the preceding problem for abelian groups. We use additive notation for abelian groups which is the case for the remainder of this paper. The first author asked whether cyclic groups are strongly sequenceable in 2000. Bode and Harborth [9] showed that the answer is yes for the cyclic group $Z_{n}$ whenever the the sum of the elements in the connection set $S$ is not 0 and either $|S|=n-1$ or $|S|=n-2$.

The same problem was discovered independently by Archdeacon, also restricted to cyclic groups, and studied in [8]. The authors prove that all cyclic groups of order at most 25 are strongly sequenceable. They also show that there is an orthogonal directed path or orthogonal directed cycle whenever the connection set $S$ has cardinality at most 6 for all cyclic groups..

Costa, Morini, Pasotti and Pellegrini [10] observed that almost all the methods employed for the previously cited work do not depend on the group being cyclic. Consequently, their paper deals with abelian groups. They use computer verification to show that all abelian groups of orders at most 23 are strongly sequenceable. They also look at the problem in terms of the cardinality of $S$ but with two restrictions, namely, they do not allow 
$S$ to contain any inverse pairs, that is, if $g \in S$, then $-g \notin S$, and they insist the elements sum to 0 . With these restrictions in place, they show that if $|S| \leq 9$, the Cayley digraph admits either an orthogonal directed cycle.

In some new work, Hicks, Ollis and Schmitt [15] restrict themselves to the case that the group has prime order. They improve the Bode and Harborth result to include $|S|=p-3$, and they improve the cardinality of the connection set result to $|S| \leq 10$. Thus, a circulant digraph of prime order admits an orthogonal directed path or an orthogonal directed cycle whenever its out-valency (and in-valency) is at most 10.

There is one obvious fact about a Cayley digraph on an abelian group we now observe. For the connection set $S$, let $\Sigma S$ denote the sum of the elements in $S$.

Proposition 2.1. Let $\vec{X}=\overrightarrow{\mathrm{Cay}}(G ; S)$ be a Cayley digraph on an abelian group $G$. When $\vec{X}$ admits an orthogonal directed cycle or directed path $\vec{Y}$, then $\vec{Y}$ is a directed cycle if $\Sigma S=0$; otherwise, it is a directed path.

Proof. If we use one arc of each length $s \in S$ and we start at vertex $g$, the directed trail formed terminates at $g+\Sigma S$ no matter in which order we choose the lengths because $G$ is abelian. From this it is easy to see that the proposition follows.

\section{The associated poset}

We use $\subseteq$ for subset inclusion so that $A \subset B$ means that $A$ is a proper subset of $B$.

We define a poset $\mathcal{P}$ to be diffuse if the following properties hold:

- The elements of $\mathcal{P}$ are subsets of a ground set $\Omega$ and the order relation is set inclusion;

- $\emptyset \in \mathcal{P}$;

- Every non-empty element of $\mathcal{P}$ has cardinality at least 2;

- If $A, B \in \mathcal{P}$ are disjoint, then $A \cup B \in \mathcal{P}$;

- If $A, B \in \mathcal{P}$ and $A \subset B$, then $B \backslash A \in \mathcal{P}$; and

- If $A, B \in \mathcal{P}$ and $A$ and $B$ are not comparable, then $|A \triangle B| \geq 3$.

In order to simplify the discussion, if the ground set has cardinality at least 1 and the empty set is the only element in the poset, we shall say this poset is diffuse.

Definition 3.1. Let $\vec{X}=\overrightarrow{\mathrm{Cay}}(G ; S)$ be a Cayley digraph on the abelian group $G$. The associated poset $\mathcal{P}(\vec{X})$ is defined as follows. The ground set is $S$ and and the elements are any non-empty subsets $S^{\prime}$ of $S$ such that $\Sigma S^{\prime}=0$ plus the empty set.

Theorem 3.2. If $\vec{X}=\overrightarrow{\mathrm{Cay}}(G ; S)$ is a Cayley digraph on the abelian group $G$, then the associated poset $\mathcal{P}(\vec{X})$ is diffuse.

Proof. If $S^{\prime}$ is a non-empty subset of $G \backslash\{0\}$ whose elements sum to 0 , then clearly $S^{\prime}$ has at least two elements of $S$. If $S^{\prime}, S^{\prime \prime} \in \mathcal{P}(\vec{X})$, then the sum of the elements in each of the subsets is 0 . If the two subsets are disjoint, then the sum of the elements in their union also is 0 implying that $S^{\prime} \cup S^{\prime \prime} \in \mathcal{P}(\vec{X})$. If $S^{\prime \prime} \subset S^{\prime}$ and both belong to $\mathcal{P}(\vec{X})$, then 
clearly the elements of $S^{\prime} \backslash S^{\prime \prime}$ also sum to 0 . This implies $S^{\prime} \backslash S^{\prime \prime} \in \mathcal{P}(\vec{X})$. Finally, if $S^{\prime}, S^{\prime \prime} \in \mathcal{P}(\vec{X})$ and they are not comparable, there must be at least one element of $S^{\prime}$ not in $S^{\prime \prime}$ and vice versa. If the symmetric difference $S^{\prime} \triangle S^{\prime \prime}$ has exactly two elements $x, y \in S$, then $x=y$ would hold because $S$ is a subset of an abelian group. This is a contradiction and the conclusion follows.

Given a sequence $s_{1}, s_{2}, \ldots, s_{n}$, a segment denotes a subsequence of consecutive entries. The notation $\left[s_{i}, s_{j}\right]$ is used for the segment $s_{i}, s_{i+1}, \ldots, s_{j}$, where $i \leq j$.

Definition 3.3. Let $\mathcal{P}$ be a poset on a groundset $\Omega=\left\{s_{1}, s_{2}, \ldots, s_{k}\right\}$ with set inclusion as the order relation. We say that $\mathcal{P}$ is sequenceable if there is a sequence $a_{1}, a_{2}, \ldots, a_{k}$ of all the elements of $\Omega$ such that no proper segment of the sequence is an element of $\mathcal{P}$. The sequence is called an admissible sequence.

We only require that proper segments are not elements of $\mathcal{P}$ in the preceding definition because we wish to allow all of $\Omega$ to be an element of the poset and still have the poset possibly be sequenceable.

Corollary 3.4. Let $\vec{X}=\overrightarrow{\mathrm{Cay}}(G ; S)$ be a Cayley digraph on the abelian group $G$. If the associated poset $\mathcal{P}(\vec{X})$ is sequenceable, then $\vec{X}$ admits either an orthogonal directed path or an orthogonal directed cycle.

Proof. Let $s_{1}, s_{2}, \ldots, s_{k}$ be an admissible sequence for $\mathcal{P}(\vec{X})$. If we take a directed trail of arcs of lengths $s_{1}, s_{2}, \ldots, s_{k}$ in that order, it is easy to see that we obtain an orthogonal directed path of length $k$ if $\Sigma S \neq 0$, whereas, we obtain an orthogonal directed cycle of length $k$ when $\Sigma S=0$.

Conjecture 3.5. Diffuse posets are sequenceable.

Because of Theorem 3.2 and Corollary 3.4, the truth of Conjecture 3.5 would imply that abelian groups are strongly sequenceable. We do not prove the conjecture here, but it does shift the work to looking at a restricted family of posets and getting away from the structure of the groups.

\section{The poset approach}

Recall that an atom in a poset is an element that covers a minimal element of the poset. When the empty set is an element, it is the unique minimal element so that the atoms are the sets not containing any non-empty proper subset in the poset. As we are considering posets whose elements are sets, we shall refer to an atom of cardinality $t$ as a $t$-atom. Because of the properties possessed by diffuse posets, once we have a list of the atoms we know all the elements of the poset. The elements are all possible unions of mutually disjoint atoms. Note that the same element may arise in more than one way as a union of atoms.

Given a poset $\mathcal{P}$ whose elements are subsets of a ground set $\Omega$, then the poset induced on a subset $\Omega^{\prime} \subseteq \Omega$ is the collection of all members of $\mathcal{P}$ that lie entirely in $\Omega^{\prime}$. This poset is denoted by $\mathcal{P}\left\langle\Omega^{\prime}\right\rangle$. Note that an induced subposet of a diffuse poset is itself diffuse.

Lemma 4.1. If every atom of a diffuse poset $\mathcal{P}$ is a 2-atom, then $\mathcal{P}$ is sequenceable. 
Proof. The 2-atoms of $\mathcal{P}$ are mutually disjoint because $\mathcal{P}$ is diffuse. Let $\left\{a_{1}, b_{1}\right\},\left\{a_{2}, b_{2}\right\}$, $\ldots,\left\{a_{t}, b_{t}\right\}$ be the 2 -atoms of $\mathcal{P}$, and let $x_{1}, x_{2}, \ldots, x_{r}$ be any elements not belonging to atoms. Note that none of the elements $x_{1}, x_{2}, \ldots, x_{r}$ belong to any element of $\mathcal{P}$ because its non-empty elements are disjoint unions of atoms. The sequence

$$
a_{1}, a_{2}, \ldots, a_{t}, x_{1}, x_{2}, \ldots, x_{r}, b_{1}, b_{2}, \ldots, b_{t}
$$

is admissible and the proof is complete.

We shall use the language of a segment belonging or not belonging to a diffuse poset $\mathcal{P}$ and this refers to the set of elements in the segment belonging to $\mathcal{P}$.

Lemma 4.2. Let $\mathcal{P}$ be a diffuse poset with ground set $\Omega$. If $\Omega \in \mathcal{P}$ and every diffuse poset on a ground set of cardinality $|\Omega|-1$ is sequenceable, then for each $s \in \Omega$, there is an admissible sequence whose first term is $s$.

Proof. Let $s$ be any element of the ground set $\Omega$. The set $\Omega \backslash\{s\}$ does not belong to the induced poset $\mathcal{P}^{\prime}=\mathcal{P}\langle\Omega \backslash\{s\}\rangle$ because $\Omega \in \mathcal{P}$. The poset $\mathcal{P}^{\prime}$ is diffuse and has an admissible sequence $a_{1}, a_{2}, \ldots, a_{t}$ by hypothesis. We claim the sequence $s, a_{1}, a_{2}, \ldots, a_{t}$ is admissible for $\mathcal{P}$.

Any proper segment of the latter sequence not containing $s$ is not in $\mathcal{P}$ because $\mathcal{P}^{\prime}$ is an induced poset. If there is a proper subsequence containing $s$ belonging to $\mathcal{P}$, then by complementation and the fact that $\Omega \in \mathcal{P}$, the rest of the sequence belongs to $\mathcal{P}$. But this contradicts the fact that the sequence $a_{1}, a_{2}, \ldots, a_{t}$ is admissible for $\mathcal{P}^{\prime}$. This concludes the proof.

Lemma 4.3. Let $\mathcal{P}$ be a diffuse poset with ground set $\Omega$. If there exists an element $s \in \Omega$ such that $\Omega \backslash\{s\} \in \mathcal{P}$, s belongs to a single atom, and all diffuse posets on ground sets of cardinality $|\Omega|-2$ are sequenceable, then $\mathcal{P}$ is sequenceable.

Proof. Let $s$ and $\mathcal{P}$ satisfy the hypotheses. Let $a_{1}$ be an element of the atom containing $s$ such that $a_{1} \neq s$. By Lemma 4.2, there is an admissible sequence $a_{1}, a_{2}, \ldots, a_{t}$ for the induced poset $\mathcal{P}\langle\Omega \backslash\{s\}\rangle$. Consider the sequence $a_{1}, a_{2}, \ldots, a_{t-1}, s, a_{t}$.

The set composed of the entire sequence is not in $\mathcal{P}$ because the latter is diffuse. Any segment not containing $s$ is not in $\mathcal{P}$ as the segment is part of an admissible sequence for $\mathcal{P}\langle\Omega \backslash\{s\}\rangle$. Thus, if there is a proper segment in $\mathcal{P}$, it must contain $s$ which implies it must contain $a_{1}$ because $s$ belongs to only one atom. But the segment $\left[a_{1}, s\right]$ cannot be in $\mathcal{P}$ because the cardinality of the symmetric difference of $\left[a_{1}, s\right]$ and $\Omega \backslash\{s\}$ is 2 . The result follows.

Lemma 4.4. Let $\mathcal{P}$ be a diffuse poset with ground set $\Omega$, where $|\Omega| \geq 3$. If there exists an element $s \in \Omega$ such that $\Omega \backslash\{s\}$ is an atom, then $\mathcal{P}$ is sequenceable.

Proof. If $s$ belongs to no atoms, then any sequence of the elements of $\Omega$ such that $s$ is at neither end is admissible. The preceding is the case when $|\Omega|=3$. If $s$ belongs only to a single atom $A$, then there must be $x, y \in \Omega$ such that $x, y \in \Omega \backslash A$ by the symmetric difference condition. It is straightforward to verify that any sequence beginning $x, s, y$ is admissible by observing that neither $x$ nor $y$ can be in the atom $A$. Thus, we may assume $s$ belongs to at least two atoms. 
Hence, we have that $|\Omega|>4$ and $s$ belongs to an $r$-atom $A$ with $r \geq 3$ because an element belongs to at most one 2-atom. Choose $A$ so that $r$ is maximum among all atoms containing $s$. Note that $|A|<|\Omega|-1$ because $\mathcal{P}$ is diffuse. Let the elements of $A$ be $s, s_{2}, \ldots, s_{r}$ and let $y \neq s$ be an element of $\Omega$ not belonging to $A$.

We claim the sequence $\pi=s_{2}, s, s_{3}, \ldots, s_{r-1}, y, s_{r}, \ldots$ completed by any permutation of the remaining elements is admissible for $\mathcal{P}$. To verify this, first observe that no segment beginning from the third entry or later belongs to $\mathcal{P}$ because the entries form a proper subset of $\Omega \backslash\{s\}$ which is an atom. The elements of the entire sequence do not belong to $\mathcal{P}$ because the poset is diffuse.

Any segment of the form $\left[s_{2}, x\right]$, where $x \in\left\{s, s_{3}, \ldots, s_{r-1}\right\}$, is a proper subset of $A$ so that it does not belong to $\mathcal{P}$. The segment $\left[s_{2}, y\right]$ does not belong to $\mathcal{P}$ because the symmetric difference with $A$ has cardinality 2 . Finally, any segment of the form $\left[s_{2}, x\right]$, where $x$ is any element from $s_{r}$ or later in $\pi$, cannot be an atom because this contradicts the choice of $A$. Thus, if it is in $\mathcal{P}$, there would be an atom properly contained in $\Omega \backslash\{s\}$.

The only segments remaining to check are those beginning with $s$. The argument for these is essentially the same as for those beginning with $s_{2}$. One difference is the segment $\left[s, s_{r}\right]$ but it has cardinality 2 symmetric difference with $A$ so cannot be in $\mathcal{P}$. Another difference is the segment $[s, y]$. If this segment is in $\mathcal{P}$, then interchange $s_{r-1}$ and $s_{r}$ in the sequence and the new segment $[s, y]$ cannot be in $\mathcal{P}$ because of the symmetric difference condition. The switching argument just used requires that $r \geq 4$, and when this holds the rest of the argument is the same as the preceding paragraph completing the proof.

When $r=3$, the sequence $\pi$ begins $s_{2}, s, y, s_{3}$. The segment $[s, y] \in \mathcal{P}$ implies that $\{s, y\}$ is a 2-atom. However, there are at least two elements of $\Omega \backslash\{s\}$ not in $A$. So choose one that does not form a 2-atom with $s$.

Lemma 4.5. Let $\mathcal{P}$ be a diffuse poset with ground set $\Omega$, where $|\Omega| \geq 4$. If there exist $s_{1}, s_{2} \in \Omega$ such that $\Omega \backslash\left\{s_{1}, s_{2}\right\}$ is an atom, then $\mathcal{P}$ is sequenceable.

Proof. If $|\Omega|=4$, then either $\left\{s_{1}, s_{2}\right\}$ also is a 2 -atom in which case $\mathcal{P}$ is sequenceable by Lemma 4.1, or neither $s_{1}$ nor $s_{2}$ are in atoms in which case $\mathcal{P}$ is easily seen to be sequenceable. If $|\Omega|=5$, then either $\left\{s_{1}, s_{2}\right\}$ is a 2-atom, just one of $s_{1}, s_{2}$ belongs to a 2-atom, both $s_{1}, s_{2}$ belong to 2-atoms, neither $s_{1}$ nor $s_{2}$ belong to a 2-atom, $s_{1}, s_{2}$ belong to a 3 -atom or $s_{1}, s_{2}$ belong to a 4 -atom. It is easy to find an admissible sequence in all six situations.

We assume $|\Omega|>5$ for the rest of the proof. Let $A$ denote the atom $\Omega \backslash\left\{s_{1}, s_{2}\right\}$. Let $M\left(s_{1}\right), M\left(s_{2}\right)$ and $M\left(s_{1}, s_{2}\right)$ denote the collections of atoms containing $s_{1}$ and not $s_{2}, s_{2}$ and not $s_{1}$, and both $s_{1}, s_{2}$, respectively. We assume that at least one of $M\left(s_{1}\right)$ and $M\left(s_{2}\right)$ contains a $k$-atom for $k \geq 3$ as it is easy to verify that $\mathcal{P}$ is sequenceable when this is not the case.

Given an atom $A_{1}$ in $M\left(s_{1}\right)$ of maximum cardinality $r+1, r \geq 2$, stretching the atom refers to a sequence of the form $a_{1}, s_{1}, a_{2}, \ldots, a_{r-1}, x, a_{r}$, where $x$ is an element to be named later and $a_{1}, a_{2}, \ldots, a_{r}$ is any sequence of the distinct elements of $A_{1}$ different from $s_{1}$. Let $B=A \backslash A_{1}=\left\{b_{1}, b_{2}, \ldots, b_{q}\right\}$ and note that $q \geq 2$ because $\mathcal{P}$ is diffuse. We first consider the case $M\left(s_{2}\right)=\emptyset$.

Start a sequence $\pi$ by stretching the atom $A_{1}$ and choose $x=b_{1}$. Complete the sequence as $a_{r}, b_{2}, b_{3}, \ldots, b_{q}, s_{2}$. We now verify that $\pi$ is admissible.

Because $M\left(s_{2}\right)$ is empty, the only possible proper segment ending with $s_{2}$ that can be in $\mathcal{P}$ is $\left[s_{1}, s_{2}\right]$. If it is in $\mathcal{P}$, then it must be an atom and the theorem holds by Lemma 4.4. 
So we consider only segments not ending with $s_{2}$.

Any such segment beginning with an element different from $a_{1}$ or $s_{1}$ is a proper subset of $A$ which implies it is not in $\mathcal{P}$. Almost all proper segments beginning with $a_{1}$ or $s_{1}$ are not in $\mathcal{P}$ because they either are proper subsets of $A_{1}$ or violate the maximality of $\left|A_{1}\right|$. The exceptional segments are $\left[a_{1}, b_{1}\right],\left[s_{1}, a_{r}\right]$ and $\left[s_{1}, b_{1}\right]$. The segments $\left[a_{1}, b_{1}\right]$ and $\left[s_{1}, a_{r}\right]$ are not in $\mathcal{P}$ because the symmetric difference with $A_{1}$ has cardinality 2 in both cases. If $\left[s_{1}, b_{1}\right] \in \mathcal{P}$, then interchange $b_{1}$ and $b_{2}$ in $\pi$. The resulting sequence is then admissible.

Thus, we now consider the case that $M\left(s_{2}\right) \neq \emptyset$. Because both $M\left(s_{1}\right)$ and $M\left(s_{2}\right)$ are non-empty, we may assume that no atom in $M\left(s_{2}\right)$ has cardinality bigger than $\left|A_{1}\right|$. Over all atoms in $M\left(s_{2}\right)$, let $\ell$ be the largest cardinality of the intersections with $B$. Let $A_{2}$ be an atom of $M\left(s_{2}\right)$ of maxium cardinality intersecting $B$ in $\ell$ elements.

Partition $A$ into four subsets as follows:

- $B_{1}=A_{1} \backslash A_{2}$;

- $B_{2}=A_{1} \cap A_{2}$;

- $B_{3}=B \backslash A_{2} ;$ and

- $B_{4}=B \cap A_{2}$.

We present the argument for the case $B_{2}=B_{3}=\emptyset$ in detail and use it to dispose of the remaining cases fairly quickly. In this case we see that the atoms $A_{1}$ and $A_{2}$ are disjoint and $A_{1} \cup A_{2}=\Omega$. This implies that $\Omega \in \mathcal{P}$ which, in turn, implies that $\left\{s_{1}, s_{2}\right\}$ is a 2-atom.

Using the same notation for the elements as above, define the sequence

$$
\pi=a_{1}, s_{1}, a_{2}, \ldots, a_{r-1}, b_{1}, a_{r}, b_{2}, \ldots, b_{q}, s_{2} .
$$

We use $\pi$ to find an admissible sequence.

Consider segments of the form $\left[a_{1}, x\right]$. If $x \in\left\{s_{1}, a_{2}, \ldots, a_{r-1}\right\}$, then $\left[a_{1}, x\right]$ is not in $\mathcal{P}$ because it is a proper subset of the atom $A_{1}$. If $x=b_{1}$, then the symmetric difference of $A_{1}$ and $\left[a_{1}, b_{1}\right]$ has cardinality 2 which implies $\left[a_{1}, b_{1}\right] \notin \mathcal{P}$.

For $x \in\left\{a_{r}, b_{2}, \ldots, b_{q}\right\}$, if $\left[a_{1}, x\right] \in \mathcal{P}$, then because the segment contains the elements of $A_{1}$, the elements of the segment not belonging to $A_{1}$ would have to be in $\mathcal{P}$. But this is impossible because they form a proper subset of $A_{2}$. Hence, no proper segment beginning with $a_{1}$ belongs to $\mathcal{P}$.

We move to segments beginning with $s_{1}$, that is, of the form $\left[s_{1}, x\right]$. If $x \in\left\{a_{2}, a_{3}, \ldots\right.$, $\left.a_{r-1}\right\}$, then it is a proper subset of $A_{1}$ so that it is not in $\mathcal{P}$. If $\left[s_{1}, b_{1}\right] \in \mathcal{P}$, then interchange $b_{1}$ and $b_{2}$ (and their labels too). The new interval $\left[s_{1}, b_{1}\right]$ does not belong to $\mathcal{P}$. This interchange is possible because $q \geq 2$ as noted earlier.

The interval $\left[s_{1}, a_{r}\right]$ cannot belong to $\mathcal{P}$ because the cardinality of the symmetric difference with $A_{1}$ is 2 . If $x \in\left\{b_{2}, b_{3}, \ldots, b_{q}\right\}$, the interval $\left[s_{1}, x\right]$ is not an atom because it has cardinality bigger than $\left|A_{1}\right|$. But the elements of the interval not belonging to an atom containing $s_{1}$ form a proper subset of $A$ and cannot belong to $\mathcal{P}$. Finally, the interval $\left[s_{1}, s_{2}\right]$ is not in $\mathcal{P}$ because $\Omega \in \mathcal{P}$.

Of the remaining intervals, the only ones which are not proper subsets of $A$ are those ending in $s_{2}$ so that we now examine intervals of the form $\left[y, s_{2}\right]$. Any such interval belonging to $\mathcal{P}$ must be an atom otherwise the elements of the segment not in the atom containing 
$s_{2}$ is a proper subset of $A$. Thus, when $y \in\left\{b_{2}, b_{3}, \ldots, b_{q}\right\},\left[y, s_{2}\right]$ is not in $\mathcal{P}$ because it is a proper subset of $A_{2}$.

The interval $\left[a_{r}, s_{2}\right]$ is not in $\mathcal{P}$ because the cardinality of the symmetric difference with $A_{2}$ is 2 . The intervals $\left[y, s_{2}\right]$, for $y \in\left\{a_{2}, a_{3}, \ldots, a_{r-1}, b_{1}\right\}$, are not in $\mathcal{P}$ as they would contradict the choice of $A_{2}$.

We see that most intervals are trivially eliminated as possible elements of $\mathcal{P}$ in the preceding argument. There are several crucial intervals and they are all we discuss in the remaining cases.

Now let $B_{2}=\emptyset$ and $B_{3} \neq \emptyset$. Moreover, label the elements of $B$ so that $B_{3}=$ $\left\{b_{1}, b_{2}, \ldots, b_{q-\ell}\right\}$ and $B_{4}=\left\{b_{q-\ell+1}, \ldots, b_{q}\right\}$. We modify the sequence $\pi$ slightly depending on the value of $q-\ell$. Here are the three scenarios. When $q-\ell=1$, let $\pi$ be the same through $a_{r-1}$ and end the sequence as

$$
a_{r-1}, b_{2}, a_{r}, b_{1}, b_{3}, \ldots, b_{q}, s_{2} \text {. }
$$

When $q-\ell=2$, end the sequence as

$$
a_{r-1}, b_{1}, a_{r}, b_{3}, b_{2}, b_{4}, \ldots, b_{q}, s_{2} .
$$

When $q-\ell>2$, end the sequence as

$$
a_{r-1}, b_{1}, a_{r}, b_{2}, \ldots, b_{q-\ell-1}, b_{q-\ell+1}, b_{q-\ell}, b_{q-\ell+2}, \ldots, s_{2} .
$$

First consider segments of the form $\left[y, s_{2}\right]$ for $y \notin\left\{a_{1}, s_{1}\right\}$ and for all three scenarios. The interval $\left[b_{q-\ell}, s_{2}\right]$ has symmetric difference of cardinality 2 with $A_{2}$ so is not in $\mathcal{P}$. The interval $\left[y, s_{2}\right]$ for $y \in\left\{b_{q-\ell+2}, \ldots, b_{q}\right\}$ is a proper subset of $A_{2}$ implying it is not in $\mathcal{P}$.

The interval $\left[b_{q-\ell+1}, s_{2}\right]$ has bigger intersection with $B$ than $A_{2}$ so that it cannot be an atom. This implies it is not in $\mathcal{P}$ as this would imply the existence of an atom properly contained in $A$. We obtain essentially the same contradiction for all other values of $y$ distinct from $a_{1}$ and $s_{1}$. Notice that these intervals are eliminated independent of the choice of $a_{r} \in A_{1} \backslash\left\{s_{1}\right\}$.

No segment of the form $\left[a_{1}, x\right]$ belongs to $\mathcal{P}$ in any of the three preceding scenarios for the same reasons discussed earlier. This conclusion holds independent of the choice of $a_{1} \in A_{1} \backslash\left\{s_{1}\right\}$. The only problematic segments beginning with $s_{1}$ are $\left[s_{1}, s_{2}\right],\left[s_{1}, b_{2}\right]$ in the first scenario, and $\left[s_{1}, b_{1}\right]$ in the second and third scenarios.

If both $\left[s_{1}, s_{2}\right]$ and $\left[s_{1}, b_{2}\right]$, or both $\left[s_{1}, s_{2}\right]$ and $\left[s_{1}, b_{1}\right]$ are in $\mathcal{P}$, then interchanging $a_{1}$ and $a_{2}$ results in an admissible sequence. If just $\left[s_{1}, s_{2}\right] \in \mathcal{P}$, then interchange $a_{1}$ and $a_{r}$ to obtain an admiisible sequence. Finally, if just $\left[s_{1}, b_{2}\right]$ or $\left[s_{1}, b_{1}\right]$ belongs to $\mathcal{P}$, then interchange $a_{r-1}$ and $a_{r}$ to obtain an admissible sequence.

The preceding interchanges require $r \geq 3$ to hold so we consider the special subcase $r=2$ separately. This case means that $A$ is a 3 -atom $\left\{s_{1}, a_{1}, a_{2}\right\}$. If $s_{2}$ is in a 3-atom $\left\{s_{2}, b_{q-1}, b_{q}\right\}$ and $b_{1}, \ldots, b_{q-2}$ are the remaining elements of $B$, then $a_{1}, s_{1}, b_{q-1}, a_{2}, b_{q-2}$, $b_{q-3}, \ldots, b_{1}, b_{q}, s_{2}$ is an admissible sequence. When $s_{2}$ is not in a 3 -atom, then it is easy to find an admissible sequence.

Now we examine the case that $B_{2} \neq \emptyset$ and $B_{3}=\emptyset$. Label the elements of $A_{1}$ so that $B_{1}=\left\{a_{1}, a_{2}, \ldots, a_{t}\right\}$ and $B_{2}=\left\{a_{t+1}, a_{t+2}, \ldots, a_{r}\right\}$. Note that $t \geq 2$ because $A_{2}$ contains all of $B$ and $\left|A_{2}\right| \leq\left|A_{1}\right|$. When $t \geq 3$, modify the original sequence $\pi$ by 
interchanging the positions of $a_{t}$ and $a_{t+1}$. The previous arguments for segments beginning with $a_{1}$ and $s_{1}$ are valid and we look at segments of the form $\left[y, s_{2}\right]$. The interval $\left[a_{t}, s_{2}\right]$ has symmetric difference of cardinality 2 with $A_{2}$ so it is not in $\mathcal{P}$.

When $t=2, A_{1}=\left\{s_{1}, a_{1}, a_{2}, \ldots, a_{r}\right\}$ and $A_{2}=\left\{s_{2}, b_{1}, b_{2}, a_{3}, \ldots, a_{r}\right\}$. The sequence $a_{1}, s_{1}, a_{3}, a_{2}, \ldots, a_{r-1}, b_{1}, a_{r}, b_{2}, s_{2}$ has only $\left[s_{1}, b_{1}\right]$ and $\left[s_{1}, s_{2}\right]$ as possible inadmissible segments. If both are inadmissible, then interchanging $a_{1}$ and $a_{2}$ produces an admissible sequence. If just $\left[s_{1}, b_{1}\right]$ is inadmissible, then interchanging $b_{1}$ and $b_{2}$ does the job. If just $\left[s_{1}, s_{2}\right]$ is inadmissible, then interchange $a_{1}$ and $a_{2}$ making the new $\left[s_{1}, s_{2}\right]$ admissible. If the new $\left[s_{1}, b_{1}\right]$ still is admissible, then we are done. However, if the new $\left[s_{1}, b_{1}\right]$ is inadmissible, then interchanging $b_{1}$ and $b_{2}$ finally achieves an admissible sequence.

The preceding argument works when $r \geq 2$. When $r=1$, the sequence $a_{1}, s_{1}, a_{3}$, $b_{1}, a_{2}, b_{2}, s_{2}$ has only the segments $\left[s_{1}, b_{1}\right],\left[a_{2}, s_{2}\right]$ and $\left[s_{1}, s_{2}\right]$ that may be inadmissible. When the 6 -segment is inadmissible, it either is a 6 -atom or there is a unique partition into two 3 -atoms. If it is a 6 -atom, there is an admissible sequence by Lemma 4.4. In the other situation, it is a straightforward, though tedious, exercise to find an interchange of elements that achieves an admissible sequence for the various partitions into two 3-atoms.

When the 6-segment is admissible, it is easy to fix any problems with the two 3segments. This completes this case.

We now consider the final case that both $B_{2}$ and $B_{3}$ are non-empty. We first provide a general argument and then examine any special cases arising because certain $B_{i}$ sets are too small.

Label elements of $B$ as: $B_{3}=\left\{b_{1}, \ldots, b_{q-\ell}\right\}$ and $B_{4}=\left\{b_{q-\ell+1}, \ldots, b_{q}\right\}$. Consider the sequence

$$
\pi=a_{1}, s_{1}, a_{2}, \ldots, a_{r-1}, b_{1}, a_{r}, b_{2}, \ldots, b_{q-\ell-1}, b_{q-\ell+1}, b_{q-\ell}, \ldots, b_{q}, s_{2} .
$$

Proper segments beginning with $a_{1}$ do not belong to $\mathcal{P}$ for the same reasons given earlier. Segments of the form $\left[s_{1}, y\right], y \notin\left\{b_{1}, s_{2}\right\}$, fail to be in $\mathcal{P}$ for the same reasons as before.

If the segment $\left[s_{1}, s_{2}\right] \in \mathcal{P}$, then we may assume it is not an atom as Lemma 4.4 implies $\mathcal{P}$ is sequenceable otherwise. Then $\left[s_{1}, s_{2}\right]$ is not a disjoint union of three or more atoms because this would give an atom properly contained in $A$. Hence, because the segment contains $A_{2},\left[s_{1}, b_{q-\ell-1}\right] \cup\left\{b_{q-\ell}\right\}$ must be an atom. But the cardinality of the latter set of elements either has cardinality bigger than $A_{1}$ or has symmetric difference with $A_{1}$ of cardinality 2 . In either case we see that it cannot be an atom. Thus, $\left[s_{1}, s_{2}\right]$ is not in $\mathcal{P}$.

The segment $\left[s_{1}, b_{1}\right]$ could belong to $\mathcal{P}$ and if it does, this is fixed by interchanging $a_{1}$ and $a_{2}$. This results in an admissible sequence. Now we consider situations for parameters being too small to let $\pi$ breathe.

The proof requires $r \geq 3$ in order to make all segments beginning with $a_{1}$ not be members of $\mathcal{P}$. Because $r>1$, we are considering $r=2$ which means $A_{1}=\left\{s_{1}, a_{1}, a_{2}\right\}$. So we begin a sequence with $a_{1}, s_{1}$ but now cannot use $a_{2}$ as the next element. Because $B_{2}$ is non-empty and $\left|A_{2}\right| \leq\left|A_{1}\right|$, we may assume $A_{2}=\left\{s_{1}, a_{2}\right\}$ or $\left\{s_{2}, a_{2}, b_{q}\right\}$.

We need to make certain the sequence does not end with the elements of $A_{2}$. We know that $B_{3} \neq \emptyset$. If it has at least two elements, then we may choose $b_{1}$ so that $\left\{s_{1}, b_{1}\right\}$ is not an atom. In this case, we start the sequence $a_{1}, s_{1}, b_{1}, a_{2}$, . The completion then depends on $q$. When $q \geq 4$, we complete the sequence so that it ends $b_{q}, b_{q-1}, s_{2}$. The sequence is admissible independent of whether or not $b_{q} \in A_{2}$. 
There several other cases to check for $1 \leq q \leq 3$ and $\left|B_{3}\right|=1$ and they can be checked similarly. This completes the proof.

\section{Small cardinality posets}

There are several items worth mentioning before stating the main theorem. First, when discussing a sequenceable poset, we tacitly assume the order relation is set inclusion for subsets of a ground set $\Omega$. Second, we now use lower case letters from the beginning of the alphabet for the elements of $\Omega$.

Third, it is clear that if a poset $P$ is sequenceable, then any subposet is sequenceable as well as any order-isomorphic poset which has arisen via a permutation of $\Omega$. The latter comment means we may relabel elements for some of the subsequent conclusions.

Theorem 5.1. If $\mathcal{P}$ is a diffuse poset whose ground set has cardinality at most 9 , then $\mathcal{P}$ is sequenceable.

Proof. It is easy to see that a diffuse poset whose ground set has cardinality 1 or 2 is sequenceable. If the ground set is $\{a, b, c\}$, then a diffuse poset has either no elements, a single 2 -atom or a single 3 -atom. The sequence $a, c, b$ is admissible assuming the 2 -atom is $\{a, b\}$ when there is a single 2 -atom.

If the ground set is $\{a, b, c, d\}$, then $\mathcal{P}$ is sequenceable if $\{a, b, c, d\}$ belongs to $\mathcal{P}$ by Lemma 4.2 , in particular, when there are two 2 -atoms. If there is a 3 -atom, then there is an admissible sequence by Lemma 4.4. The situation is trivial if there is a single 2 -atom or no atoms at all.

So we see that diffuse posets with ground sets of cardinality at most 4 are sequenceable. We next consider ground sets of cardinality 5 .

If $\Omega=\{a, b, c, d, e\}$ and $\Omega$ belongs to the diffuse poset $\mathcal{P}$, then $\mathcal{P}$ is sequenceable by Lemma 4.2. If $\Omega \notin \mathcal{P}$ but there is an atom of cardinality 4 , then $\mathcal{P}$ is sequenceble by Lemma 4.4. If there are no 4 -atoms but there is a 3 -atom, then $\mathcal{P}$ is sequenceable by Lemma 4.5. If the only atoms are 2 -atoms, then $\mathcal{P}$ is sequenceable by Lemma 4.1. Hence, all diffuse posets with ground sets of cardinality 5 are sequenceable.

This takes us to ground sets of cardinality 6. Let $\Omega=\{a, b, c, d, e, f\}$. As before, Lemma 4.4 implies $\mathcal{P}$ is sequenceable if there is a 5 -atom and Lemma 4.5 implies $\mathcal{P}$ is sequenceable when there is a 4 -atom. Lemma 4.2 implies that a diffuse poset $\mathcal{P}$ with ground set $\Omega$ is sequenceable whenever $\Omega \in \mathcal{P}$. Thus, we may assume that every atom is either a 2 -atom or a 3 -atom and there are neither two disjoint 3 -atoms nor three 2 -atoms.

It is not difficult to verify that to within order-isomorphism there is a unique maximal diffuse poset on $\Omega$ with only 2 - and 3 -atoms, that is, every diffuse poset with this restriction on the atoms is order-isomorphic to a subposet. The atoms of this unique poset are $\{b e, c d, a b c, a d e, b d f, c e f\}$ and an admissible sequence is $d, b, c, f, a, e$.

For ground sets of cardinalities 7 and 8, the results are displayed in Tables 1 and 2 in the appendix. We have verified the result for ground sets of cardinality 9 , but the number of pages to display the table is about 30 and we have chosen to not include the table. This concludes the proof.

Corollary 5.2. Let $\vec{X}$ be a Cayley digraph on an abelian group. If the connection $S$ set for $\vec{X}$ has at most nine elements, then $\vec{X}$ admits an orthogonal directed path when $\Sigma S \neq 0$ or an orthogonal directed cycle when $\Sigma S=0$. 


\title{
ORCID iDs
}

\author{
Brian Alspach (D) https://orcid.org/0000-0002-1034-3993 \\ Georgina Liversidge (D) https://orcid.org/0000-0002-4467-4328
}

\section{References}

[1] B. Alspach, D. L. Kreher and A. Pastine, The Friedlander-Gordon-Miller conjecture is true, Australas. J. Combin. 67 (2017), 11-24, https://ajc.maths.uq.edu.au/?page= get_volumes\&volume $=67$.

[2] B. A. Anderson, Sequencings and starters, Pacific J. Math. 64 (1976), 17-24, http:// projecteuclid.org/euclid.pjm/1102867209.

[3] B. A. Anderson, A fast method for sequencing low order nonabelian groups, in: Combinatorial design theory, North-Holland, Amsterdam, volume 149 of North-Holland Math. Stud., pp. 27 41, 1987, doi:10.1016/S0304-0208(08)72874-3.

[4] B. A. Anderson, $S_{5}, A_{5}$ and all nonabelian groups of order 32 are sequenceable, volume 58, pp. 53-68, 1987, eighteenth Southeastern International Conference on Combinatorics, Graph Theory, and Computing (Boca Raton, Fla., 1987).

[5] B. A. Anderson, Sequencings of dicyclic groups, Ars Combin. 23 (1987), 131-142.

[6] B. A. Anderson, Sequencings of dicyclic groups. II, J. Combin. Math. Combin. Comput. 3 (1988), 5-27.

[7] B. A. Anderson, All dicyclic groups of order at least twelve have symmetric sequencings, in: Finite geometries and combinatorial designs (Lincoln, NE, 1987), Amer. Math. Soc., Providence, RI, volume 111 of Contemp. Math., pp. 5-21, 1990, doi:10.1090/conm/111/1079734.

[8] D. S. Archdeacon, J. H. Dinitz, A. Mattern and D. R. Stinson, On partial sums in cyclic groups, J. Combin. Math. Combin. Comput. 98 (2016), 327-342.

[9] J.-P. Bode and H. Harborth, Directed paths of diagonals within polygons, Discrete Math. 299 (2005), 3-10, doi:10.1016/j.disc.2005.05.006.

[10] S. Costa, F. Morini, A. Pasotti and M. A. Pellegrini, A problem on partial sums in abelian groups, Discrete Math. 341 (2018), 705-712, doi:10.1016/j.disc.2017.11.013.

[11] J. Dénes and A. D. Keedwell, Latin squares, volume 46 of Annals of Discrete Mathematics, North-Holland Publishing Co., Amsterdam, 1991, new developments in the theory and applications, With contributions by G. B. Belyavskaya, A. E. Brouwer, T. Evans, K. Heinrich, C. C. Lindner and D. A. Preece, With a foreword by Paul Erdős, https://www.sciencedirect.com/bookseries/ annals-of-discrete-mathematics/vol/46/suppl/C.

[12] R. J. Friedlander, B. Gordon and M. D. Miller, On a group sequencing problem of Ringel, in: Proceedings of the Ninth Southeastern Conference on Combinatorics, Graph Theory, and Computing (Florida Atlantic Univ., Boca Raton, Fla., 1978), Utilitas Math., Winnipeg, Man., Congress. Numer., XXI, 1978 pp. 307-321.

[13] B. Gordon, Sequences in groups with distinct partial products, Pacific J. Math. 11 (1961), 1309-1313, http://projecteuclid.org/euclid.pjm/1103036916.

[14] P. Headley, $R$-sequenceability and $R^{*}$-sequenceability of abelian 2-groups, Discrete Math. 131 (1994), 345-350, doi:10.1016/0012-365X(94)90396-4. 
[15] J. Hicks, M. A. Ollis and J. R. Schmitt, Distinct partial sums in cyclic groups: polynomial method and constructive approaches, J. Combin. Des. 27 (2019), 369-385, doi:10.1002/jcd. 21652.

[16] G. B. Hoghton and A. D. Keedwell, On the sequenceability of dihedral groups, in: Algebraic and geometric combinatorics, North-Holland, Amsterdam, volume 65 of North-Holland Math. Stud., pp. 253-258, 1982, doi:10.1016/S0304-0208(08)73270-5.

[17] M. A. Ollis, Sequenceable groups and related topics, Electron. J. Combin. (2013), \#DS10v2, doi:10.37236/30.

[18] G. Ringel, Cyclic arrangements of the elements of a group, Notices Amer. Math. Soc. 21 (1974), A95-96, https://www.ams.org/cgi-bin/notices/amsnotices. plethispage=collectionnav\&article_id=fullissue\&article_type= gallery\&gallery_type=fullissue\&gallery_volume=1974. 


\section{Appendix}

Table 1 below provides admissible sequences for all diffuse posets with a ground set of cardinality 7 and Table 2 does the same for ground sets of cardinality 8 . However, two conventions are the following:

(1) No poset having the ground set as an element is included because they are sequenceable by Lemma 4.2; and

(2) only posets with $k$-atoms for $k \in\{2,3,4\}$ and $k \in\{2,3,4,5\}$ are listed in Tables 1 and 2 , respectively, as the others are sequenceable by the lemmas of Section 4.

\begin{tabular}{|c|c|c|}
\hline included atoms & excluded atoms & sequence \\
\hline ab,cd,ef,ace,adg & $\mathrm{cfg}$ & b,d,a,e,g,c,f \\
\hline ab,cd,ef,ace & adg,bdeg & $\mathrm{a}, \mathrm{c}, \mathrm{b}, \mathrm{e}, \mathrm{d}, \mathrm{g}, \mathrm{f}$ \\
\hline ab,cd,ef,acg & ade,adf,adfg & $\mathrm{b}, \mathrm{e}, \mathrm{d}, \mathrm{a}, \mathrm{f}, \mathrm{g}, \mathrm{c}$ \\
\hline $\mathrm{ab}, \mathrm{cd}, \mathrm{ef}$ & xyz,acfg & $\mathrm{a}, \mathrm{c}, \mathrm{g}, \mathrm{f}, \mathrm{d}, \mathrm{e}, \mathrm{b}$ \\
\hline ab cd,aef,ceg,bdf & xy & $\mathrm{c}, \mathrm{a}, \mathrm{e}, \mathrm{b}, \mathrm{d}, \mathrm{g}, \mathrm{f}$ \\
\hline ab,cd,aef,ceg & xy,bdf & $a, d, f, e, g, b, c$ \\
\hline ab,cd,aef,bce & xy,cfg,deg,dfg & $\mathrm{a}, \mathrm{d}, \mathrm{f}, \mathrm{c}, \mathrm{g}, \mathrm{e}, \mathrm{b}$ \\
\hline $\mathrm{ab}, \mathrm{cd}, \mathrm{aef}$ & xy,ceg,bce,acfg & e,b,d,a,f,c,g \\
\hline ab,cd,ace & xy,bef,afg,bfg,adg & $\mathrm{b}, \mathrm{d}, \mathrm{a}, \mathrm{g}, \mathrm{c}, \mathrm{e}, \mathrm{f}$ \\
\hline $\mathrm{ab}, \mathrm{cd}$ & xy,xyz,bcef & $\mathrm{e}, \mathrm{b}, \mathrm{f}, \mathrm{c}, \mathrm{a}, \mathrm{d}, \mathrm{g}$ \\
\hline ab,acd,cef,beg & xy & a,d,g,e,f,b,c \\
\hline $\mathrm{ab}, \mathrm{acd}, \mathrm{cef}$ & xy,beg,acfg & $b, d, a, f, c, g, e$ \\
\hline ab,acd,bef & xy,ceg,acfg & a,c,g,f,d,e,b \\
\hline ab,acd,bce & xy,def,cfg,dfg,bfg,bdef & $\mathrm{a}, \mathrm{f}, \mathrm{b}, \mathrm{e}, \mathrm{d}, \mathrm{c}, \mathrm{g}$ \\
\hline $\mathrm{ab}, \mathrm{acd}$ & xy,cef,bef,bce,adef & e,d,f,a,c,b,g \\
\hline $\mathrm{ab}, \mathrm{cde}$ & xy,axy,bxy,acef,acdg & b,f,e,a,c,d,g \\
\hline ab,acde & xy,xyz & b,c,f,a,d,e,g \\
\hline $\mathrm{ab}$ & xy,xyz,axyz,bxyz,defg & a,d,e,b,f,g,c \\
\hline abc,ade,bdf & xy,bdeg & $\mathrm{c}, \mathrm{a}, \mathrm{e}, \mathrm{b}, \mathrm{d}, \mathrm{g}, \mathrm{f}$ \\
\hline abc,ade,abdf & xy,bef,cef,bdg,beg,ceg & $\mathrm{a}, \mathrm{c}, \mathrm{g}, \mathrm{b}, \mathrm{f}, \mathrm{d}, \mathrm{e}$ \\
\hline abc,ade & xy,bdf,abdf & $\mathrm{f}, \mathrm{b}, \mathrm{d}, \mathrm{a}, \mathrm{c}, \mathrm{e}, \mathrm{g}$ \\
\hline abc,def & $\begin{array}{c}\text { xy,xyz,abeg,abfg,acdg } \\
\text { bcdg,adeg,adfg,bdeg,bdfg }\end{array}$ & $\mathrm{a}, \mathrm{c}, \mathrm{g}, \mathrm{d}, \mathrm{b}, \mathrm{e}, \mathrm{f}$ \\
\hline abc,abde & $x y, x y z$ & $\mathrm{c}, \mathrm{a}, \mathrm{f}, \mathrm{b}, \mathrm{d}, \mathrm{e}, \mathrm{g}$ \\
\hline $\mathrm{abc}$ & xy,xyz,abxy,acxy,bcxy & a,d,e,b,c,f,g \\
\hline abcd & $x y, x y z$,acef & $\mathrm{b}, \mathrm{g}, \mathrm{d}, \mathrm{a}, \mathrm{c}, \mathrm{e}, \mathrm{f}$ \\
\hline
\end{tabular}

Table 1: Ground set of cardinality 7

We alter the notation for the tables in two ways. First, we use words rather than set notation because it saves considerable space. Second, we use roman letters rather than italics because the appearance of the words is better. In summary, the atom $\{a, b, c\}$ in the main body of the paper appears as abc in the tables.

The tables have been compacted to an extent that makes it necessary to describe how to read them. 
The column headed "included atoms" contains a list of atoms that definitely belong to the poset under discussion. The only convention to keep in mind here is that an entry in parentheses - such as in row 12 in Table 1-indicates that precisely one of the words is an atom but not both. So in this situation, one of bf or $\mathrm{cg}$ is a 2-atom but it is not the case that both 2 -atoms are in the poset.

The column headed "excluded atoms" indicates which atoms are definitely not in the poset, but there are some conventions being followed. These conventions are now listed.

\begin{tabular}{|c|c|l|} 
included atoms & excluded atoms & sequence \\
\hline ab,cd,ef,acg,beh & - & a,d,g,c,e,b,f,h \\
\hline ab,cd,ef,acg,beg & bfh,deh,dfh & a,h,c,g,e,d,f,b \\
\hline ab,cd,ef,acg,bgh & deh,deg & c,h,a,g,e,b,f,d \\
\hline ab,cd,ef,acg,egh & bfh,bfg,cfh & a,g,e,c,h,f,d,b \\
\hline ab,cd,ef,acg & adf,beh,beg,bgh,egh & b,d,a,f,c,g,h,e \\
\hline ab,cd,ef,agh,ace & gxy,hxy & c,a,d,g,f,b,e,h \\
\hline ab,cd,ef,agh & xyz,acfg & d,b,e,a,g,f,c,h \\
\hline ab,cd,ef,ace,bdf & xyz & a,c,b,g,d,f,h,e \\
\hline ab,cd,ef & xyz,acfg & b,d,c,a,f,d,e,h \\
\hline ab,cd,aef,bcg,egh & xy & a,d,e,f,g,h,c,b \\
\hline ab,cd,aef,bcg & xy,egh & g,e,b,c,f,d,a,h \\
\hline ab,cd,aef,egh & xy,bcg,bcf & a,d,f,e,g,c,h,b \\
\hline ab,cd,aef,ceg & $\begin{array}{c}\text { xy,bdg,bch } \\
\text { bdh,fgh,beh }\end{array}$ & e,h,b,g,c,a,d,f \\
\hline
\end{tabular}

Table 2: Ground set of cardinality 8 (Continued)

(1) Any atom that violates the definition of a diffuse poset because of an included atom, certainly is not in the poset and it is not listed as an excluded atom. For example, if $a b c$ is an included atom, then no other 3-atom may contain ab, ac or bc so they simply are not listed in the excluded atoms column. Similarly, if abcd is an included atom, then neither abce nor abc can be atoms and they are not listed in the excluded atoms column.

(2) If an excluded atom uses letters from the ground set, then that particular atom is not in the poset.

(3) If an excluded atom uses letters from the end of the alphabet, then it means that all atoms of that cardinality different from any included atoms are excluded. For example, in line 7 of Table 1, xy indicates that there are no 2 -atoms other than ae, and xyzw indicates that abcd is the only 4 -atom in the poset.

(4) If an excluded atom uses both letters from the ground set and the end of the alphabet, it means all atoms of that form different from any included atoms are not in the poset. For example, in poset 51 of Table 2, ab is an included atom and both axy and bxy are excluded. This means there are no 3 -atoms containing a and no 3 -atoms containing b. Of course, there are no 3 -atoms containing both a and b because ab is a 2-atom.

(5) Excluded atoms in boldface indicate all atoms that can be formed via label changes allowed because of the included atoms are excluded. The following example should 


\begin{tabular}{|c|c|c|}
\hline included atoms & excluded atoms & sequence \\
\hline $\mathrm{ab}, \mathrm{cd}, \mathrm{aef}$ & xy,bcg,egh,ceg,acfg & e,g,c,f,a,d,b,h \\
\hline ab,cd,ace,efg & xy,afg,afh,bef,beh,bfg,bfh & $\mathrm{a}, \mathrm{e}, \mathrm{f}, \mathrm{c}, \mathrm{h}, \mathrm{d}, \mathrm{g}, \mathrm{b}$ \\
\hline ab,cd,ace & xy,afg,bef,bfg,efg,adh & $\mathrm{f}, \mathrm{b}, \mathrm{d}, \mathrm{a}, \mathrm{h}, \mathrm{c}, \mathrm{e}, \mathrm{g}$ \\
\hline ab,cd,efg,aefh & $\mathrm{xy}, \mathrm{xyz}$ & $\mathrm{c}, \mathrm{d}, \mathrm{h}, \mathrm{a}, \mathrm{e}, \mathrm{d}, \mathrm{f}, \mathrm{g}$ \\
\hline ab,cd,efg & $\begin{array}{c}\text { xy,xyz,aefh,adef,befh,cefh } \\
\text { aegh,afgh,begh,bfgh,defh } \\
\text { cegh,cfgh,degh,dfgh,adef }\end{array}$ & $\mathrm{b}, \mathrm{e}, \mathrm{a}, \mathrm{d}, \mathrm{f}, \mathrm{c}, \mathrm{h}, \mathrm{g}$ \\
\hline ab,cd,aefg & $\mathrm{xy}, \mathrm{xyz}$ & $\mathrm{e}, \mathrm{b}, \mathrm{f}, \mathrm{a}, \mathrm{c}, \mathrm{g}, \mathrm{d}, \mathrm{h}$ \\
\hline $\mathrm{ab}, \mathrm{cd}$ & xy,xyz,aefg & $\mathrm{e}, \mathrm{b}, \mathrm{g}, \mathrm{a}, \mathrm{c}, \mathrm{h}, \mathrm{d}, \mathrm{f}$ \\
\hline ab,acd,bef,ceg,agh & xy,dfg & $\mathrm{c}, \mathrm{e}, \mathrm{h}, \mathrm{f}, \mathrm{b}, \mathrm{d}, \mathrm{a}, \mathrm{g}$ \\
\hline ab,acd,bef,ceg & xy,agh & $\mathrm{a}, \mathrm{d}, \mathrm{g}, \mathrm{c}, \mathrm{f}, \mathrm{e}, \mathrm{h}, \mathrm{b}$ \\
\hline ab,acd,bef,aeg & $\mathrm{xy}, \mathbf{c f g}, \mathrm{ceh}, \mathbf{c f h}$ & $\mathrm{b}, \mathrm{g}, \mathrm{f}, \mathrm{a}, \mathrm{e}, \mathrm{c}, \mathrm{d}, \mathrm{h}$ \\
\hline ab,acd,bef,agh & xy,ceg,bcg & $\mathrm{e}, \mathrm{b}, \mathrm{c}, \mathrm{a}, \mathrm{g}, \mathrm{d}, \mathrm{h}, \mathrm{f}$ \\
\hline ab,acd,bef,cgh & xy,deg,aeg,bdg & $\mathrm{a}, \mathrm{e}, \mathrm{d}, \mathrm{c}, \mathrm{g}, \mathrm{f}, \mathrm{h}, \mathrm{b}$ \\
\hline ab,acd,bef & xy,xyz,adgh & $\mathrm{a}, \mathrm{c}, \mathrm{g}, \mathrm{d}, \mathrm{e}, \mathrm{f}, \mathrm{h}, \mathrm{b}$ \\
\hline ab,acd,cef,deg,bch & $\mathrm{xy}, \mathrm{bfg}$ & $\mathrm{a}, \mathrm{d}, \mathrm{f}, \mathrm{c}, \mathrm{h}, \mathrm{e}, \mathrm{b}, \mathrm{g}$ \\
\hline ab,acd,cef,deg & $\begin{array}{c}\text { xy,beh,bfg } \\
\text { bfh,bch,bceh }\end{array}$ & d,a,h,c,b,e,f,g \\
\hline ab,acd,cef,egh & xy,bfg,dfg,aceg & $\mathrm{b}, \mathrm{g}, \mathrm{a}, \mathrm{e}, \mathrm{c}, \mathrm{d}, \mathrm{f}, \mathrm{h}$ \\
\hline ab,acd,cef,aeg & $\begin{array}{c}\text { xy,bfg,beh,bfh,bgh } \\
\text { dfg,deh,dfh }\end{array}$ & $\mathrm{b}, \mathrm{g}, \mathrm{f}, \mathrm{a}, \mathrm{e}, \mathrm{c}, \mathrm{d}, \mathrm{h}$ \\
\hline ab,acd,cef,bdg & xy,aeh,beh,deh,egh,aeg,bcfg & $\mathrm{d}, \mathrm{a}, \mathrm{g}, \mathrm{c}, \mathrm{b}, \mathrm{f}, \mathrm{e}, \mathrm{h}$ \\
\hline ab,acd,cef,bde & $\begin{array}{c}\text { xy,bfg,bgh,dfg,egh } \\
\text { fgh,aeg,afg,aeg,afh,bcdf }\end{array}$ & $\mathrm{g}, \mathrm{a}, \mathrm{c}, \mathrm{b}, \mathrm{d}, \mathrm{f}, \mathrm{e}, \mathrm{h}$ \\
\hline $\mathrm{ab}$,acd,cef,aceg & $\begin{array}{c}\text { xy,beg,bfg,beh,bfh,bgh,deg,dfg } \\
\text { deh,dfh,egh,fgh,afg,aeh } \\
\text { afh,bdg,bdh,bde,bdf }\end{array}$ & $\mathrm{b}, \mathrm{d}, \mathrm{a}, \mathrm{f}, \mathrm{c}, \mathrm{g}, \mathrm{e}, \mathrm{h}$ \\
\hline ab,acd,cef & $\begin{array}{c}\text { xy,beg,bgh,deg,egh,aeg,bdg } \\
\text { bde,aceg,bcfg }\end{array}$ & $\mathrm{d}, \mathrm{a}, \mathrm{g}, \mathrm{c}, \mathrm{f}, \mathrm{b}, \mathrm{e}, \mathrm{h}$ \\
\hline ab,acd,bce,afg & $\begin{array}{l}\text { xy,bfg,bfh,cgh } \\
\text { def,deh,dfg,dfh }\end{array}$ & b,d,e,a,c,f,g,h \\
\hline ab,acd,bce,efg & xy,afh,bfh,cgh,deh,dfh,adef & $\mathrm{c}, \mathrm{b}, \mathrm{d}, \mathrm{e}, \mathrm{f}, \mathrm{g}, \mathrm{h}$ \\
\hline ab,acd,bce,aef,adfg & $\begin{array}{c}\text { xy,bfg,bfh,bgh,cfg } \\
\text { deh,dfh,cgh,dgh,agh }\end{array}$ & $\mathrm{a}, \mathrm{c}, \mathrm{b}, \mathrm{h}, \mathrm{e}, \mathrm{f}, \mathrm{g}, \mathrm{d}$ \\
\hline ab,acd,bce,aef & $\begin{array}{l}\text { xy,bfg,bgh,cfg,deg,deh } \\
\text { dfg,cgh,dgh,agh,adfg }\end{array}$ & $\mathrm{b}, \mathrm{g}, \mathrm{a}, \mathrm{d}, \mathrm{f}, \mathrm{c}, \mathrm{e}, \mathrm{h}$ \\
\hline ab,acd,bce,fgh & xy,def,aef,adfg & $\mathrm{d}, \mathrm{c}, \mathrm{f}, \mathrm{e}, \mathrm{b}, \mathrm{g}, \mathrm{a}, \mathrm{h}$ \\
\hline
\end{tabular}

Table 2: Ground set of cardinality 8 (Continued) 


\begin{tabular}{|c|c|c|}
\hline included atoms & excluded atoms & sequence \\
\hline ab,acd,bce,adfg & xy,xyz & a,c,b,h,e,f,g,d \\
\hline ab,acd,bce & xy,xyz,adfg,bfgh & $\mathrm{e}, \mathrm{f}, \mathrm{b}, \mathrm{c}, \mathrm{a}, \mathrm{g}, \mathrm{d}, \mathrm{h}$ \\
\hline ab,acd,bcdg & $\begin{array}{l}\text { xy,bef,bfg,cef,def,ceh } \\
\text { deh,dfh,ceg,bce,adeg }\end{array}$ & $\mathrm{b}, \mathrm{d}, \mathrm{a}, \mathrm{g}, \mathrm{e}, \mathrm{f}, \mathrm{h}, \mathrm{c}$ \\
\hline ab,acd & $\begin{array}{l}\text { xy,bef,cef,def,ceg,cfg } \\
\text { deg,dfg,ceh,deh,dfh,cgh } \\
\text { dgh,bce,bcdg,bcdh }\end{array}$ & $\mathrm{g}, \mathrm{b}, \mathrm{c}, \mathrm{a}, \mathrm{e}, \mathrm{d}, \mathrm{f}, \mathrm{h}$ \\
\hline ab,acd,efg,acef & xy,xyz & b,e,f,a,h,c,d,g \\
\hline ab,acd,efg & xy,xyz,acef,begh & f,b,e,a,c,g,d,h \\
\hline ab,acd,acef & $x y, x y z$ & $\mathrm{~b}, \mathrm{~d}, \mathrm{a}, \mathrm{g}, \mathrm{c}, \mathrm{e}, \mathrm{f}, \mathrm{h}$ \\
\hline ab,acd,bcef & xy,xyz,adef,aceg,adeg,acgh,adgh & $\mathrm{d}, \mathrm{g}, \mathrm{c}, \mathrm{a}, \mathrm{e}, \mathrm{b}, \mathrm{f}, \mathrm{h}$ \\
\hline ab,acd,cefg & $\begin{array}{c}\text { xy,xyz,acef,adef,aceh,adeh } \\
\text { bceh,bdef,bcef,bdeh,bcgh,begh }\end{array}$ & $\mathrm{b}, \mathrm{f}, \mathrm{a}, \mathrm{d}, \mathrm{e}, \mathrm{c}, \mathrm{g}, \mathrm{h}$ \\
\hline ab,acd & xy,xyz,acef,bcef,cefg,befh,begh & f,b,e,a,c,h,d,g \\
\hline ab,cde,cfg,dfh,acef & xy,axy,bxy & $\mathrm{b}, \mathrm{d}, \mathrm{a}, \mathrm{e}, \mathrm{f}, \mathrm{h}, \mathrm{c}, \mathrm{g}$ \\
\hline ab,cde,cfg,dfh & xy,axy,bxy,acef,bcdf & e,a,d,b,f,c,h,g \\
\hline ab,cde,cfg,acdf & $\mathrm{xy}, \mathrm{xyz}$ & $\mathrm{b}, \mathrm{h}, \mathrm{d}, \mathrm{a}, \mathrm{c}, \mathrm{e}, \mathrm{f}, \mathrm{g}$ \\
\hline $\mathrm{ab}, \mathrm{cde}, \mathrm{cfg}$ & $x y, x y z$, acdf,bfgh & d,a,e,c,f,b,g,h \\
\hline ab,cde,acdf & xy,xyz,aceh & b,e,a,h,c,d,f,g \\
\hline ab,cde,acfg & $\begin{array}{l}\text { xy,xyz,adef,bcdf,bdef } \\
\text { acdh,adeh,bcdh,bdeh }\end{array}$ & $\mathrm{b}, \mathrm{d}, \mathrm{a}, \mathrm{e}, \mathrm{f}, \mathrm{c}, \mathrm{g}, \mathrm{h}$ \\
\hline ab,cde & xy,xyz,acdf,acfg,cefg & f,a,g,c,b,e,d,h \\
\hline ab,acde,bcdf & $\mathrm{xy}, \mathrm{xyz}$ & a,e,g,c,d,f,h,b \\
\hline $\mathrm{ab}, \mathrm{acde}, \mathrm{acfg}$ & $\begin{array}{c}\text { xy,xyz,bcdf,bdef } \\
\text { bdeg,bcdh,bceh,bdeh }\end{array}$ & $\mathrm{b}, \mathrm{d}, \mathrm{a}, \mathrm{e}, \mathrm{f}, \mathrm{c}, \mathrm{g}, \mathrm{h}$ \\
\hline ab,acde & $\begin{array}{l}\text { xy,xyz,bcdf,acfg } \\
\text { acefg,acdfh,acdgh }\end{array}$ & $\mathrm{b}, \mathrm{e}, \mathrm{f}, \mathrm{c}, \mathrm{a}, \mathrm{d}, \mathrm{h}, \mathrm{g}$ \\
\hline ab,cdef & xy,xyz,axyz,bxyz,acdeh,acdfg & $b, g, f, a, c, d, e, h$ \\
\hline $\mathrm{ab}$,acdef & xy,xyz,xyzw & b,c,g,a,d,e,f,h \\
\hline$a b$ & xy,xyz,xyzw,axyzw,defgh & $\mathrm{a}, \mathrm{d}, \mathrm{e}, \mathrm{b}, \mathrm{f}, \mathrm{g}, \mathrm{h}, \mathrm{c}$ \\
\hline abc,ade,bdf,afg,beh & xy,bcfg & $\mathrm{c}, \mathrm{b}, \mathrm{d}, \mathrm{a}, \mathrm{f}, \mathrm{e}, \mathrm{g}, \mathrm{h}$ \\
\hline abc,ade,bdf,afg,ceh & xy,bgh,dgh,cdef & $\mathrm{b}, \mathrm{a}, \mathrm{d}, \mathrm{c}, \mathrm{e}, \mathrm{f}, \mathrm{h}, \mathrm{g}$ \\
\hline $\begin{array}{l}\text { abc, ade,bdf } \\
\text { afg,bcfh }\end{array}$ & $\begin{array}{l}\text { xy,beh,cdh,efh,bgh } \\
\text { dgh,ceh,cgh,egh }\end{array}$ & $\mathrm{a}, \mathrm{c}, \mathrm{g}, \mathrm{f}, \mathrm{b}, \mathrm{h}, \mathrm{d}, \mathrm{e}$ \\
\hline abc,ade,bdf,afg & $\begin{array}{c}\text { xy,beh } \\
\text { cgh,bcfh,bdeh,dfgh }\end{array}$ & c,a,e,b,d,h,f,g \\
\hline
\end{tabular}

Table 2: Ground set of cardinality 8 (Continued) 


\begin{tabular}{|c|c|c|}
\hline included atoms & excluded atoms & sequence \\
\hline abc,ade,bdf,ceg,cfh & xy,afg,bcgh & a,f,d,e,g,h,c,b \\
\hline abc,ade,bdf,ceg,bfgh & $\begin{array}{l}\text { xy,afg,afh,beh } \\
\text { cdh,cfh,efh,dgh }\end{array}$ & $\mathrm{a}, \mathrm{b}, \mathrm{f}, \mathrm{c}, \mathrm{g}, \mathrm{h}, \mathrm{e}, \mathrm{d}$ \\
\hline abc,ade,bdf,ceg & $\begin{array}{l}\text { xy,afg,afh,beh } \\
\text { cfh,bfgh,acfg }\end{array}$ & $\mathrm{b}, \mathrm{f}, \mathrm{c}, \mathrm{g}, \mathrm{a}, \mathrm{e}, \mathrm{h}, \mathrm{d}$ \\
\hline abc,ade,bdf,cgh,abeg & xy,afg,afh,beh,efg,efh & a,b,d,e,g,f,h,c \\
\hline abc,ade,bdf,cgh,acdg & $\begin{array}{l}\text { xy,afg,beg,beh,afh,efg } \\
\text { efh,abeg,abeh,abfh,abfg }\end{array}$ & $\mathrm{b}, \mathrm{a}, \mathrm{f}, \mathrm{c}, \mathrm{d}, \mathrm{g}, \mathrm{h}, \mathrm{e}$ \\
\hline abc,ade,bdf,cgh & $\begin{array}{l}\text { xy,afg,efg,abeg } \\
\text { acdg,bcfg }\end{array}$ & e,g,a,c,d,b,h,f \\
\hline abc,ade,bdf,agh,bcfg & $\begin{array}{l}\text { xy,beg,cdg,beh,cdh } \\
\text { ceg,efg,ceh,cfh,efh }\end{array}$ & a,c,g,b,h,f,d,e \\
\hline abc,ade,bdf,agh & $\begin{array}{l}\text { xy,beg,ceg,cfg } \\
\text { bcfg,abdg }\end{array}$ & $\mathrm{c}, \mathrm{g}, \mathrm{a}, \mathrm{b}, \mathrm{d}, \mathrm{e}, \mathrm{f}, \mathrm{h}$ \\
\hline abc,ade,bdf,abeg & $\begin{array}{l}\text { xy,afg,cdg,afh,beh,cdh } \\
\text { ceg,cfg,efg,ceh,cfh,efh } \\
\text { cgh,egh,fgh,agh,bgh,dgh }\end{array}$ & $\mathrm{c}, \mathrm{b}, \mathrm{f}, \mathrm{a}, \mathrm{e}, \mathrm{g}, \mathrm{d}, \mathrm{h}$ \\
\hline abc,ade,bdf,acfg & $\begin{array}{c}\text { xy,beg,cdg,afh,beh,cdh } \\
\text { ceg,efg,ceh,cfh,efh,cgh } \\
\text { egh,fgh,agh,bgh,dgh,abeg } \\
\text { bcdg,bdeg,abeh,abfh } \\
\text { acdh,adfh,bcdh,bdeh }\end{array}$ & $\mathrm{b}, \mathrm{h}, \mathrm{c}, \mathrm{a}, \mathrm{g}, \mathrm{e}, \mathrm{f}, \mathrm{d}$ \\
\hline abc,ade,bdf,bcfg & $\begin{array}{c}\text { xy,beg,beh,cfh,egh,bgh } \\
\text { bfg,afg,afh,ceg,ceh,cgh } \\
\text { agh,abeg,abfg,acdg } \\
\text { aefg,acfh,bceh,abeh } \\
\text { abfh,acdh,acfg,bceg,aefh }\end{array}$ & $\mathrm{a}, \mathrm{c}, \mathrm{g}, \mathrm{b}, \mathrm{h}, \mathrm{f}, \mathrm{d}, \mathrm{e}$ \\
\hline abc,ade,bdf & $\begin{array}{c}\text { xy,afg,ceg,cgh } \\
\text { agh,abeg,acfg,bcfg,bcfh }\end{array}$ & $\mathrm{g}, \mathrm{c}, \mathrm{a}, \mathrm{d}, \mathrm{b}, \mathrm{e}, \mathrm{f}, \mathrm{h}$ \\
\hline abc,ade,bfg,dfh,cgh & xy,xyz,adfg & b,a,f,d,g,h,e,c \\
\hline abc,ade,bfg & xy,cef,cdg,ceg,beh,ceh & $\mathrm{c}, \mathrm{a}, \mathrm{d}, \mathrm{b}, \mathrm{e}, \mathrm{f}, \mathrm{h}, \mathrm{g}$ \\
\hline $\begin{array}{c}\text { abc,ade,bfg } \\
\text { dfh,acef }\end{array}$ & $\begin{array}{l}\text { xy,bdf,bef,beg,bdgcdg } \\
\text { ceg,adfg,abdg,bcdf }\end{array}$ & $\mathrm{b}, \mathrm{c}, \mathrm{g}, \mathrm{a}, \mathrm{f}, \mathrm{e}, \mathrm{d}, \mathrm{h}$ \\
\hline abc,ade,bfg,dfh & $\begin{array}{c}\text { xy,cef,cdg,ceg } \\
\text { beh,ceh,abef,acef }\end{array}$ & $\mathrm{c}, \mathrm{a}, \mathrm{h}, \mathrm{b}, \mathrm{d}, \mathrm{e}, \mathrm{f}, \mathrm{g}$ \\
\hline
\end{tabular}

Table 2: Ground set of cardinality 8 (Continued) 


\begin{tabular}{|c|c|c|}
\hline included atoms & excluded atoms & sequence \\
\hline abc,ade,bfg,bcfh & $\begin{array}{l}\text { xy,cdf,cdg,bdh } \\
\text { cdh, dfh,dgh }\end{array}$ & $\mathrm{a}, \mathrm{c}, \mathrm{d}, \mathrm{b}, \mathrm{f}, \mathrm{h}, \mathrm{g}, \mathrm{e}$ \\
\hline abc,ade,bfg,acdf & $\begin{array}{c}\text { xy,cef,cdg,ceg,bdh,beh } \\
\text { cdh,ceh,dfh,efh,dgh,egh } \\
\text { bcfh,bcgh,dfgh,aceh }\end{array}$ & $\mathrm{b}, \mathrm{c}, \mathrm{f}, \mathrm{a}, \mathrm{g}, \mathrm{d}, \mathrm{e}, \mathrm{h}$ \\
\hline abc,ade,bfg & $\begin{array}{l}\text { xy,cdf,bdh,beh,cdh,ceh } \\
\text { dfh bcfh,acdf,abeh }\end{array}$ & d,h,a,e,b,c,f,g \\
\hline abc,ade,abdf & $\begin{array}{l}\text { xy,bef,cef,beg } \\
\text { ceg,bdh,cdeg }\end{array}$ & $\mathrm{g}, \mathrm{e}, \mathrm{d}, \mathrm{c}, \mathrm{a}, \mathrm{f}, \mathrm{b}, \mathrm{h}$ \\
\hline abc,ade,bcdf & $\begin{array}{c}\text { xy,bef,bdg,beg } \\
\text { abef,abdg,abeg,aefh }\end{array}$ & $\mathrm{g}, \mathrm{b}, \mathrm{c}, \mathrm{d}, \mathrm{a}, \mathrm{f}, \mathrm{e}, \mathrm{h}$ \\
\hline abc,ade,abfg & $\begin{array}{c}\text { xy,bdf,cdf,bdh } \\
\text { cdh,acdf,abdh,acdh,bcdh } \\
\text { bcdf,bdef,cdef,bdeh,cdeh }\end{array}$ & d,f,g,a,e,b,c,h \\
\hline abc,ade & $\begin{array}{c}\text { xy,bdf,abdf,bcdf,abfg } \\
\text { bcfh,bcgh }\end{array}$ & f,b,c,d,a,h,e,g \\
\hline abc,def,abdg & xy,axy,bxy,cxy,acfh & e,g,b,f,a,c,h \\
\hline abc,def,adgh & $\begin{array}{c}\text { xy,axy,bxy,cxy,abeg } \\
\text { bcdg,bceg,abdfg }\end{array}$ & $\mathrm{b}, \mathrm{a}, \mathrm{g}, \mathrm{d}, \mathrm{f}, \mathrm{h}, \mathrm{e}, \mathrm{c}$ \\
\hline abc,def,abde & $\begin{array}{c}\text { xy,axy,bxy,cxy,egh,abfg } \\
\text { acdg,acfg,adgh,afgh,cfgh }\end{array}$ & f,d,g,a,e,b,c,h \\
\hline abc,def,abgh & xy,axy,bxy,cxy,xyzw,acdeg & $\mathrm{b}, \mathrm{a}, \mathrm{g}, \mathrm{d}, \mathrm{c}, \mathrm{e}, \mathrm{f}, \mathrm{h}$ \\
\hline abc,def & xy,axy,bxy,cxy,xyzw,abdfg & $\mathrm{c}, \mathrm{a}, \mathrm{g}, \mathrm{d}, \mathrm{b}, \mathrm{f}, \mathrm{e}, \mathrm{h}$ \\
\hline abc,abde,adefg & $\mathrm{xy}, \mathrm{xyz}$ & $\mathrm{c}, \mathrm{b}, \mathrm{f}, \mathrm{a}, \mathrm{d}, \mathrm{e}, \mathrm{h}, \mathrm{g}$ \\
\hline abc,abde & xy,xyz,adefg,cdefg & $\mathrm{c}, \mathrm{a}, \mathrm{f}, \mathrm{b}, \mathrm{d}, \mathrm{e}, \mathrm{g}, \mathrm{h}$ \\
\hline abc,adef & xy,xyz,abxy,acxy,bcxy,abdfg & $\mathrm{g}, \mathrm{d}, \mathrm{f}, \mathrm{a}, \mathrm{b}, \mathrm{e}, \mathrm{c}, \mathrm{h}$ \\
\hline abc,abdef & xy,xyz,axyz,bxyz,cxyz & $\mathrm{c}, \mathrm{a}, \mathrm{g}, \mathrm{b}, \mathrm{d}, \mathrm{e}, \mathrm{f}, \mathrm{h}$ \\
\hline $\mathrm{abc}$ & $\begin{array}{c}\text { xy,xyz,axyz,bxyz,cxyz,abxyz } \\
\text { acxyz,bcxyz,adefh,adegh } \\
\text { bdefg,cdefg }\end{array}$ & $\mathrm{b}, \mathrm{f}, \mathrm{d}, \mathrm{a}, \mathrm{e}, \mathrm{h}, \mathrm{g}, \mathrm{c}$ \\
\hline abcd,abef,abceg & $\mathrm{xy}, \mathrm{xyz}$ & $\mathrm{c}, \mathrm{d}, \mathrm{g}, \mathrm{a}, \mathrm{b}, \mathrm{e}, \mathrm{h}, \mathrm{f}$ \\
\hline abcd,abef & xy,xyz,abceg & $\mathrm{c}, \mathrm{g}, \mathrm{d}, \mathrm{a}, \mathrm{b}, \mathrm{e}, \mathrm{h}, \mathrm{f}$ \\
\hline abcd,abcef & $\begin{array}{c}\text { xy,xyz,abxy,acxy,adxy } \\
\text { bcxy,bdxy,cdxy,befg }\end{array}$ & d,a,h,c,b,e,f,g \\
\hline abcd & xy,xyz,abef,abcef & $\mathrm{a}, \mathrm{e}, \mathrm{f}, \mathrm{b}, \mathrm{c}, \mathrm{d}, \mathrm{g}$ \\
\hline abcde & xy,xyz,xyzw,abcfg & d,h,e,a,b,c,f,g \\
\hline
\end{tabular}

Table 2: Ground set of cardinality 8 
make this subtle concept clear. Start with the template obtained from the included atoms and observe which label changes are allowed. For example, consider the sixteenth entry from the end of Table 2. The included atoms are abc, ade so that the template is two 3 -atoms intersecting at a single point. The label a is fixed because it is the only point belonging to both 3 -atoms. The labels $\mathrm{b}$ and $\mathrm{c}$ may be switched because they lie in the same 3 -atom. The same holds for the labels $\mathrm{d}$ and e. Furthermore, the two sets $\{b, c\}$ and $\{d, e\}$ may be switched. Finally, the labels f,g,h may be switched with each other as they belong to neither 3 -atom. Thus, excluding the atom bdf means that all of the 3-atoms bdf, bef, cdf, cef, bdg, beg, cdg, ceg, bdh, beh, cdh and ceh are excluded. Similarly, excluding the atom abdf means all the atoms abdf, abef, acdf, acef, abdg, abeg, acdg, aceg, abdh, abeh, acdh and aceh are excluded. 